\title{
Impact of Fog and Cloud Computing Applications to Reserve a Seat in Railways
}

\author{
Gajender Kumara1, Ankush Kumar Gaurb2, Anam Khan³ \\ aComputer Science and Engineering, Shree Balwant Institute of Technology \\ Sonipat, Haryana, India \\ 1gk201719nit@gmail.com \\ 3anam.17kt000131@cse.iitism.ac \\ ${ }^{b}$ Computer Science and Engineering, Kanpur Institute of Technology \\ Kanpur, Uttar Pradesh, India \\ 2ankushkumardddm@gmail.com (Corresponding author)
}

\begin{abstract}
As India's largest employer has gone digital, this is the beginning of mass acquisition in a new dimension. Indian Railways serves on a peripheral scale in the size of each aircraft. It carries 23,000 passengers and over 3 million tonnes daily, with more than 12,000 passengers traveling at 115,000 km and 7000 stations. But as the nation's largest employer has begun to digitalize its services operations, it is beginning to gain mass in an emerging dimension. The network collects around 100 datasets per user (one truffle is enough to store 500-1000 movies). This passenger booking plate has 25 million users, leading to approximately 800,000 daily transactions. Such is the creation of a digital scale railway ecosystem, a fertile ground for entrepreneurs, employers and large scale technology for testing and testing. And while Railways has made a good start by integrating third parties with third parties, the company can create a seamless experience in booking, payment and other services at the top of the railway data pipeline. Here we have several ways to verify a passenger or passenger ticket, which will be discussed one by one on Fig. 1. We understand here that the $N$ computer system (server) is available in the availability center and the availability train provided by the NECC (North Eastern Carrying Corporation Ltd). Each seat or chair acts as a spring (Smart) chair, which means that we are connected to a ticket scanner or chair that can scan tickets and send the actual data to the field node (fog node). Later, the latest analysis will result in the proposed data and the final data that will be sent to the cloud. We are connected to multiple devices to share real-time data and updated real-time data on the cloud. We have created a fog environment and a cloud environment. We have implemented this with the help of clipping and sending data by sending real data separately. We have created a smart gateway between military (Servers) and customer (sensor), cloud and customer (sensor). In this paper, there is the solution that can enhanced the chances of getting confirm ticket, based on individual server load in coaches of the train, according to the increase in the number of sensors request, in the context of processing and considering three cases, it is an answer by analysing cloud-fog scenario. Up to this implementation the simple round robin algorithm is also used solution for load balancing purpose. The method is implemented by using a class student attendance system in a college level.
\end{abstract}

Keywords: Student Attendance, Data Pipeline, Cloud Environment, Fog Environment, Sensors.

\section{Introduction}

Indian Railways (IR) is a government owned Railway Company in India. Indian Railways recently monopolized the country's rail transportation. It is one of the largest and busiest railway networks in the world and carries more than 6 billion passengers and about 750 million tons of cargo each year. IR is the world's largest commercial or public service Employee Eastern Corporation with more than 1.6 million employees. Many public or private services have gone online. Nowadays, people can get services, interact, and get information by online. More and more Internet users 
are inspiring a lot Government-owned agencies for previous traditional changes Service in electronic standard [1].

In India or in any developed country there are already many techniques or solutions that have been implemented to solve the different problems that are occurring during reservation of the tickets for the passengers. As India's largest employer has gone digital, this is the beginning of mass acquisition in a new dimension - data. Indian Railways operates on a staggering scale in every vibratory dimension. It carries 23.3 million passengers and 3.5 million tons of cargo every day, deploying over 12,000 trains at $116,000 \mathrm{~km}$ and some 7,100 stations. But as the nation's largest employer begins to digitize parts of its services operations, it is the beginning of a mass acquisition in an emerging dimension - data. The network collects about 100 terabytes of consumer data per year. Its passenger booking platform has 25 million users, making about 800,000 daily transactions. Such a digital scale is making the railway ecosystem a fertile ground for deploying and testing entrepreneurs, innovators and large-scale technology. And while railways have made a good start by teaming up with third parties, there is a lot more to be done before companies can build a seamless experience in booking, payment and other services at the top of the railway data pipeline.

In this paper, we are giving solutions by assuming fog and cloud applications to increase the chances of confirmed tickets by using the method of a class student attendance system in a college or university. We will consider three cases and give solution. It will be discussed one by one. With this project there is a research report for reservation confirming tickets for railways.

\subsection{CHOOSING THE TRAIN ROUTE}

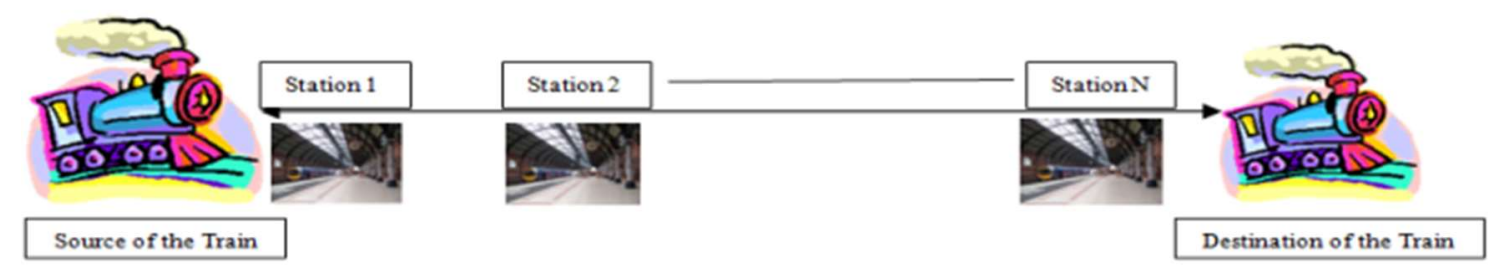

Figure 1.Routes followed by the trains

The diagram above shows the specific route for a train. It shows the number of stations that go to the source destination by railway route traffic. When booking tickets, a person can choose any boarding station. Therefore, a person has several options to choose a boarding and leaving point and can travel on their own.

\subsection{CLOUD ENVIRONMENT}

This is the latest trend in information technology. The "smart" object counts on the World Wide Web, because it does not concern the management and management of all resources. Product resources provide customers with management and management of resource environments. The customer is solely responsible for the cost of using the service and this type of service is called personnel use in cloud computing.

With this concept of cloud computing, smart devices can become the interface for big data centers. Cloud is an extended form of distributed computing environment [7], and [8]. In this paper we present the solution for increasing the chances of confirm ticket by taking quick response from the fog devices on cloud environment and analysing the data on the cloud means on the central server station.

We have implemented the load balancing algorithm with respect to response time of each fog node. After this, Section II presents fog computing based smart communication. Section III is on our work progress. After this we conclude this paper in section IV. Cloud environment is created by using cloud Google by creating VM instances, which is shown below Figure 2. 


\begin{tabular}{|c|c|c|c|c|c|c|c|}
\hline$\nabla$ Name A & Zone & Creation time & Machine type & Recommendation & Interna IP & Extema IP & Connect \\
\hline$\nabla_{1}^{\prime}$ instance & $\begin{array}{l}\text { australia- } \\
\text { southeasti-b }\end{array}$ & $\begin{array}{l}\text { 5Apr 2019, } \\
\text { 17:47:43 }\end{array}$ & $\begin{array}{l}\text { IVCPU, } 3.75 \\
G B\end{array}$ & & $\begin{array}{l}10.152 .0 .2 \\
\text { (nico) }\end{array}$ & $35.197 .162 .53 \mathrm{~L}^{\pi}$ & SSH - : \\
\hline
\end{tabular}

Figure 2. Cloud Environment

\subsection{Fog Environment}

The fog computing [6] captures steam as a modern term in the technology world, often used in contrast to cloud or mislabelled edges, two of which have topography: either the computer is on edge or the computer cloud. Is in The easiest way to understand what it is to be rational in fog. In a bright environment, sensors or devices can be everywhere, from the cloud to the cloud and anywhere in between. In some cases, you are programmed to be anti-system administration. This is not the case. Therefore, the administration that was sent to cloud today can be identified tomorrow. It is important to have a structure that affects a large biological community of assets. This processing enhances the use of assets that allow photos to be used anywhere from one location to another for benefits, which may be speed, ease of access, data transmission efficiency, Consistency and cost. The adverts have helped us come together in our daily lives and with special processing power and give you all the ability to use it for a small fee in the area, and the fist of the stars. The term computer is calculated to show different things, so if you want to take advantage of it, you have to make sure it meets all the standards. This has to be:

\section{a. Providing a range of processing power that covers the continuity of cloud conditions}

In the current cloud-based antitighting framework, a significant portion of user power is organized in the older cloud. With related components, it is estimated to achieve 20 billion by the end of two years and results in an estimated travel speed, with an increase of two years. Along these lines, devices have increased after discovering the power needed to find it, which is obtained through command entry. Edge includes a regularly rendered server, often referred to as "edge-of-thecenter", close to children to take care of severity like basic adaptive situations due to low information and information transmission is.

Because all ready data can be used to handle fraud, including PCs that are geographically most appropriate, they can also provide low latency calculations, and edge preparation can be used for unclear reasons. Is like a constant victim. Together with these lines, articulation 'edge' and 'fog' are used as synonyms as often as possible. Little attention is paid to edge counting - this is just one element of the more widespread fog count. Computer edge computing is an effective way to reduce bandwidth and bandwidth utilization of high-traffic technologies such as IP, but services provided at any point in a particular business or home vary in performance, scalability, uptime, and cost. There are different requirements that an "edge node" cannot solve.

An efficient fog computing infrastructure must be geographic to allow for edge-based computing and cloud-based cloud computing, and a range of resources ideally for flexibility and flexibility. As long as the hardware used is not just a component of a very wide spectrum of resources, it is not a fog.

\section{b. Use flawless computing resources based on interest}

The fog count not only encompasses more important geology than cloud or edge; However, that topography can be dynamic. Information about managing your computer can be located anywhere, and its area may change constantly. This is done through the administration of rationalism. For professionals sending product benefits, this means that they indicate that the administration needs to go into fog engineering, not where it will be done. For example, if low inactivity is a prerequisite, the administration will naturally be directed towards the best available 
compliance, whether it is a server in a similar room, a provincial data center, or, if nothing is faster, perhaps a Cloud Data Center.

Being able to broadly specify business conditions using fog computing can make architects' lives a lot more complex by reducing the need for provisioning, scaling, and continuous computing facilities. In certain stages of fog remediation, engineers basically require each administration to organize major events such as low latency, lost costs or environmentally friendly power consumption, so the platform will send administration to computers that demand but will meet these criteria.

\section{Foundation and Related Work}

The railroad has been spotted in many systems of ticket validation, so asking for a location is not news. A great deal of research is currently underway. Reservation is not just new to the railway. It is a flowing and old place for research for some people, in other places like aircraft, transport, etc.

Likewise, the concept of "fog" and "cloud" computing is not new these days. Many researchers have explored many uses of the fog environment in many areas. Mohammad Aazam, Sherali Zeadally, Khaled A. Harras[2] proposed that the digital world is expanding rapidly and moving into 4G Long Term Revolution (LTE), Wireless Broadband (WiBro), Low Power Broadband Network (LPWAN), 5G, Lifi and so on network services - All these have led to sophisticated services. . The number of online applications is increasing as more computing, communication, and intellectual capabilities arise.

For many applications, they required remoteness to work on behalf of the user device and return results. This is often referred to as "offloading" where tasks are outsourced and performers work together to achieve the ultimate goal of the program. Task migration is attractive to emerging loT and cloud computing applications. This can be between loT nodes, sensors, edge devices or fog nodes. Unloading can be done based on a variety of factors including program computing requirements, load balancing, power, delay management, and so on. They have recently introduced a classification scheme for loading schemes that have been proposed for areas such as fog, cloud computing and the Internet.

T. Kakagi proposed [13] that The "Reservation-Compulsory Commuting Railways" is a concept that the authors propose to eliminate in trains. Congress-free citizenship can be realized through a "reservation-mandated" policy in the railway system's ultra-high frequency functions and ability to control the behavior of passengers. Although to make the most of the capacity, the ticketing system needs to be extremely "flexible" in terms of securing and problem-solving, which is set management for travelers who are not secure in services at any given time. Can be changed. In this article, the author discusses these requirements of the ticketing system, in which, by discussing the concept of a primarily existing ticketing system and the IPASS ticketing system, the "flexibility" for understanding the mainstream railways of the reservation is by the author. Is offered. As an "ideal system" in the 1990s.

Amir Vahid Dastjerdi and Raj Kumar Buyya, University of Melbourne [3], propose The Internet of Things (IoT) may additionally require improvements to improve the quality of the genre, although it produces a wealth of information that is difficult for classic systems, the cloud, as well as its components. The Fog is designed to overcome these obstacles.

Inayat, Z. Gani, A. Anuar, N.B. Anwar, S.; Khan, M.K [4] propose Mobile Medical Computing (MCC) allows smart cellular devices (SMDs) to gain access to cloud resources, so that data can be intercepted from smart phones and gather predictive predictions for application processing. Basics solely talk about infrared assets within the MMC of IRRS and within the GMC infrastructure.

Wildlife is needed as a possible answer to potential problems. Researchers, academics, security administrators, and cloud-based services for cloud service supervision provide the framework, framework, for SMDs to work with cloud-based DRRS-assisted negotiation status., To find 
requirements and protocols. It provides complete details of IDRS in PMS MDR and HD and its importance in the birth of MCC.

\section{Research Method}

\subsection{Smart Chair or Door and Load Balancing}

\section{CONSIDERING OR PROPOSED WORK}

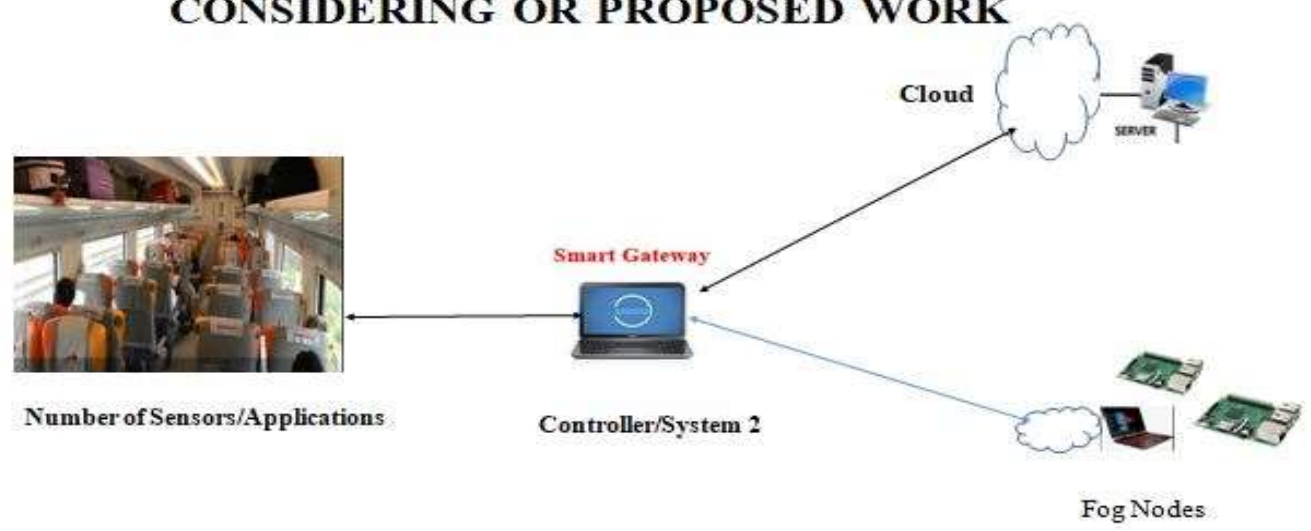

Figure 3. Load Balancing Algorithm

Creating a balance between phase calculations is an early topic that requires research and study. The data center [12] is designed with multiple systems, where balancing is an easy task especially for promotion computing. Most of the research has been done in a research environment.

The use of semi-distributed load balancing in cloud computing has not been discussed in any literature, where physical distribution is already on the list on physical computing. Using the semidistributed equilibrium method we can create a new algorithm for fog computing.

We have used some algorithms to load on a Boog server via smart gateways, as well as separate data from consecutive numbers or combinations of different routes in a smart chair.

\section{A SMART CHAIR or SMART DOOR SYSTEM}

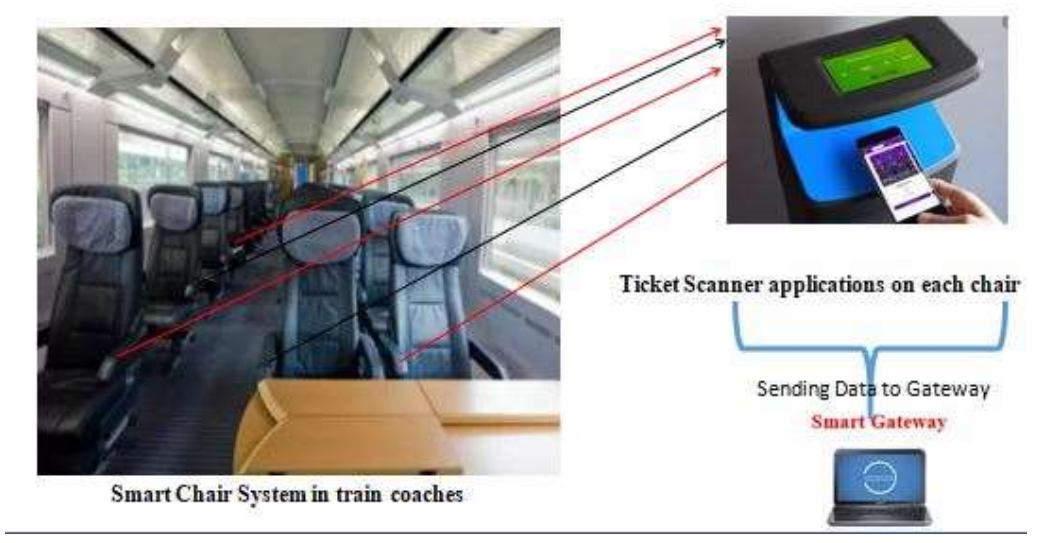

Figure 4. Smart Chairs

Figure Figure 4 above shows the smart chair or currency system. These smart chair or cars are connected or connected to a scanner ticket machine like a sensor; data is collected from these sensors and sent to the cog nodes present in each train coach. The data is now sent to the cloud or central node of each railway station's station. Send the data through a custom gateway where the balancing act works, prioritizing critical data that already indicates an analysis of the central area of the railway or we can say on the cloud. 
This paper proposes to increase the likelihood of validating a ticket, ticket for travellers, using a better weight balance for some computers, which is implemented in each central node of the cluster. Figure 3 shows the load balancing algorithm as per the implementation of the environment.

\subsection{Class Student Attendance System}

This method treats the attendance of the students present in the railway coach in the same way as the college student attendance register. When a passenger in the coach enters through the smart chair or smart door and this data is sent to the fog server and the fog server for the fog nodes, it then sends the updated data to the cloud for further analysis. The figure 3 above in section 3.1 illustrates this scenario.

\section{Result and Discussion}

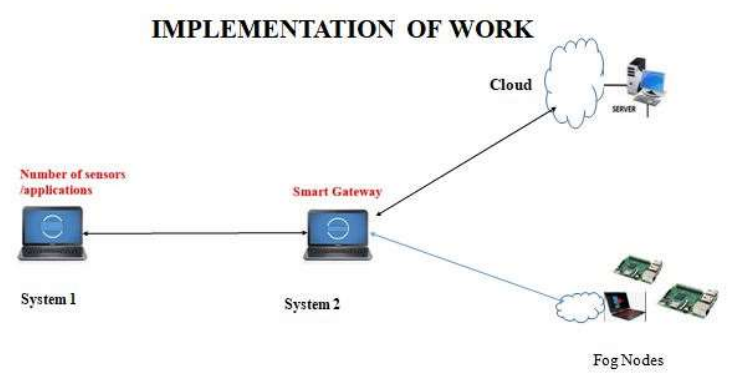

Figure 5. Implementation of the work

The work presented is to create the environment mentioned above. In this environment, System 1 displays the number of cisens or applications, while System 2 displays the smart gateway. System 1 continuously sends data to the smart gateway, the gateway line continuously transmits both cloud and military. The present result is based on actual implementation work in this paper. For this task, the Python Socket programming language is used. And cloud server is built using Google. Google Cloud SS cell is built.

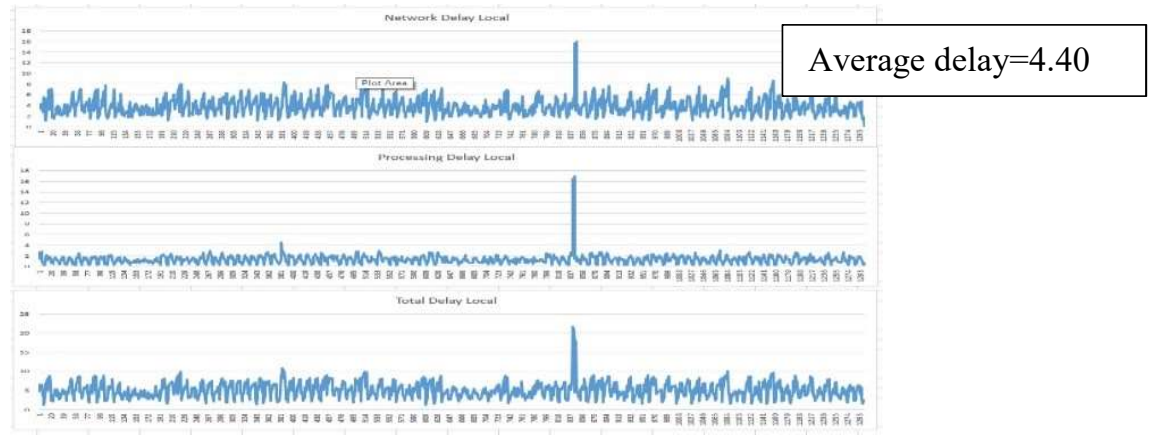

Figure 6. Network, processing, total delay in cloud environment, when bandwidth $1000 \mathrm{Mbps}$.

On seeing the Figure 6, it is conclude that there are some results i.e. network delay, processing delay and total time delay. Here vertical line shows the time taken in millisecond and horizontal line shows the continuous data of total 1025 records in excel file. In figure Figure 6 , network time is beneath 8 millisecond averagely. Processing time is below 2 millisecond averagely and total time is around 9 millisecond. When considering bandwidth 1000 Mbps. 


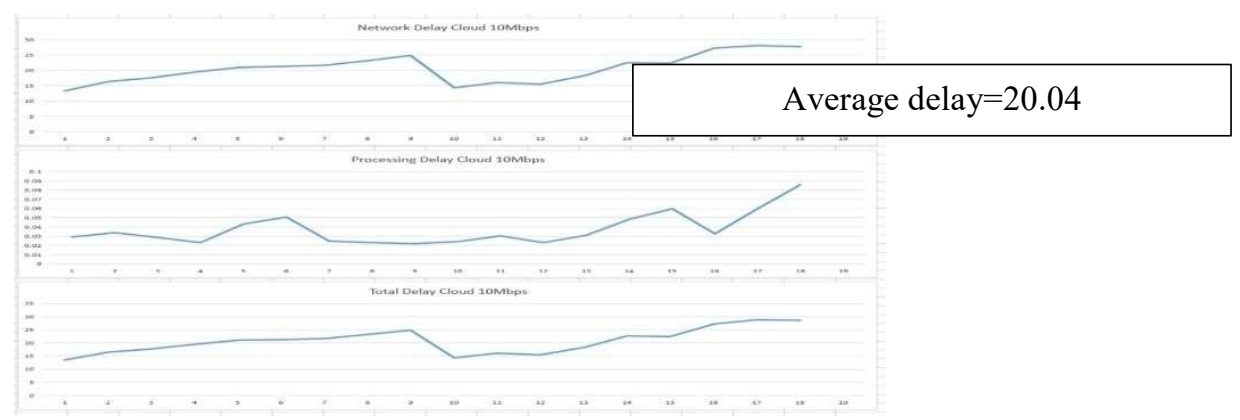

Figure 7. Network, processing, total delay in cloud when Bandwidth 10Mbps

In this Figure 7, the average network delay time is 20 milliseconds. The processing time is almost same i.e. 0.04 milliseconds. The total time is averagely 20.04 millisecond. When bandwdth condering 10 Mbps.

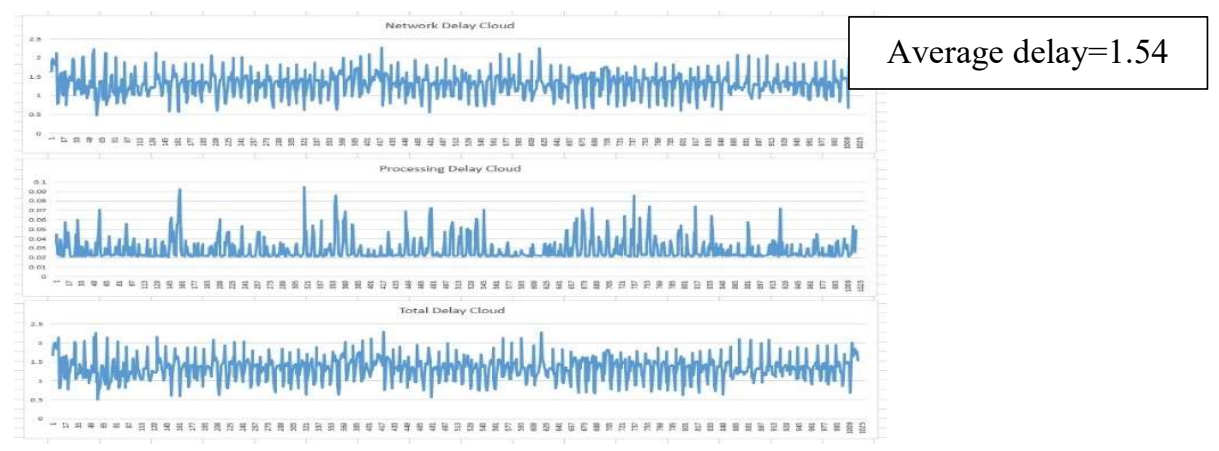

Figure 8.Network delay, processing delay and Total delay in local fog.

On analysing this Figure 8 , the time taken by the fog averagely in a network are 1.5 milliseconds while processing time in the cloud is approximately 0.04 millisecond and the total time both of them is expected 1.54 milliseconds. When considering bandwidth $1000 \mathrm{Mbps}$.

In the wake of investigating the over three graph, the conclusion is that fog is only it is the allinclusive type of cloud. Fog works same as cloud for same information, yet it relies upon some circumstance. Here the system distinction will clearly less however processing time is more than cloud, so on the off chance that it can take care of the issues at the fog level,fog processing is useful for better outcomes. For this, thereis an existing round robinalgorithm of each fog node.

Following are the some results based on real time data, by considering simple round robin algorithm and weighted round robin algorithm. Figure 8, Figure 9, and Figure 10 shows the results of simple round robin algorithm, while Figure 11, Figure 12, and Figure 13 shows the results of weighted round robin algorithm. Figure 8 shows the network delay(expected average 4.68) in simple round robin algorithm, Figure 9 shows the processing delay(expected average 0.49 ) in simple round robin algorithm, Figure 10 shows the total delay(expected average 4.69) in simple round robin algorithm, Figure 11 shows the network delay(expected average 3.54) in weighted round robin algorithm, Figure 12 shows the processing delay(expected average 0.0095) in weighted round robin algorithm, and Figure 13 shows the total delay(expected average 3.55 ) in weighted round robin algorithm.

The above results are taken by considering one local loopback server within smart gateway, one local fog node and one cloud( ssh cloud.google ) environment only. 


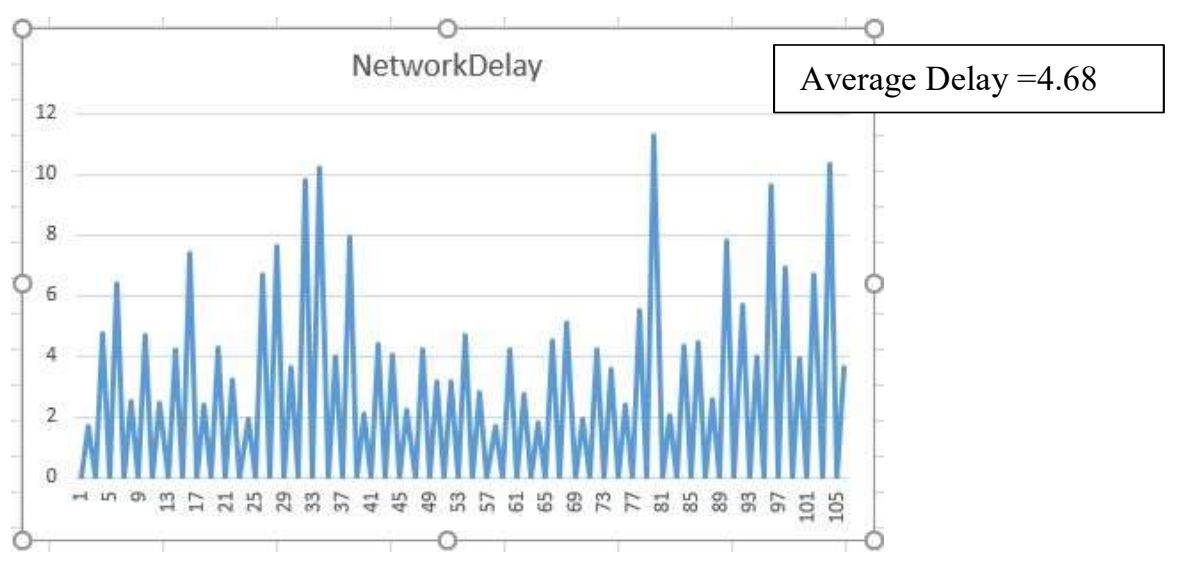

Figure 9. Network delay in simple round robin algorithm

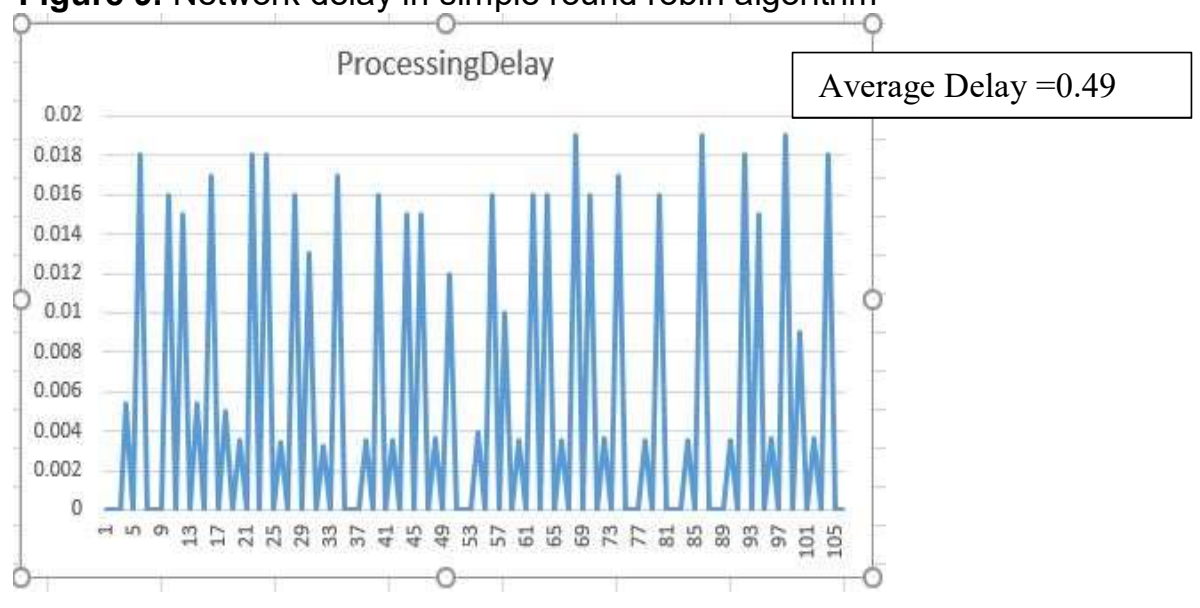

Figure 10. Processing delay in simple round robin algorithm

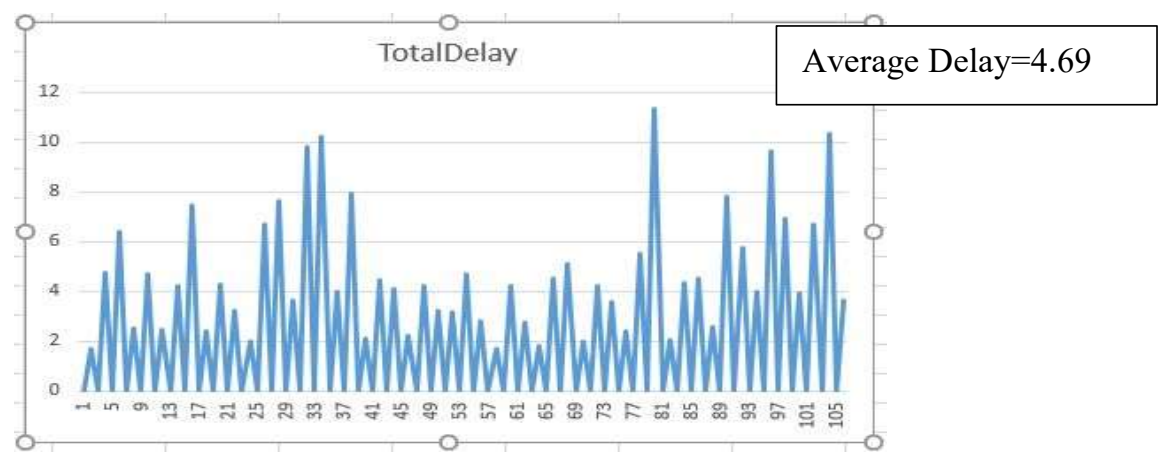

Figure 11. Total delay in simple round robin algorithm

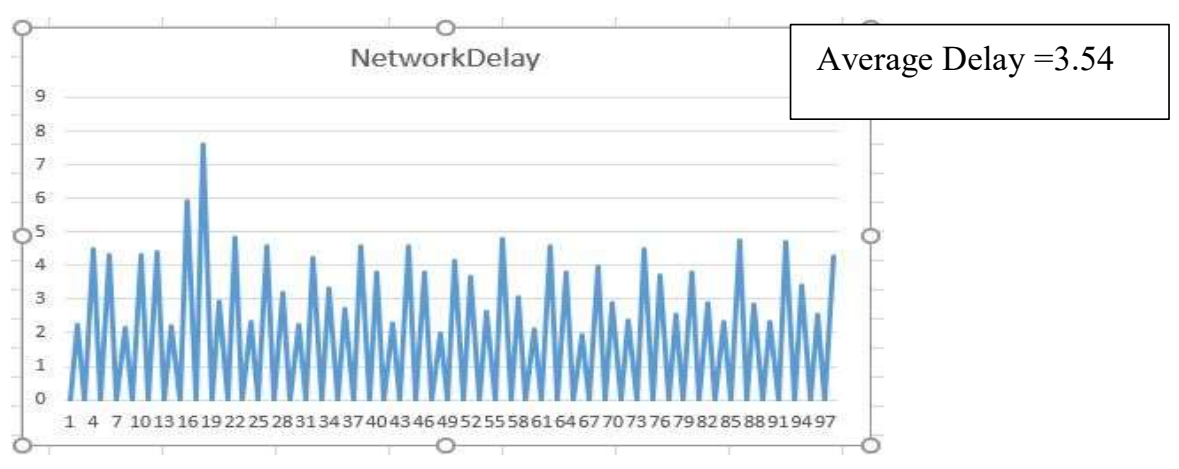


Figure 12. Network delay in weighted round robin algorithm

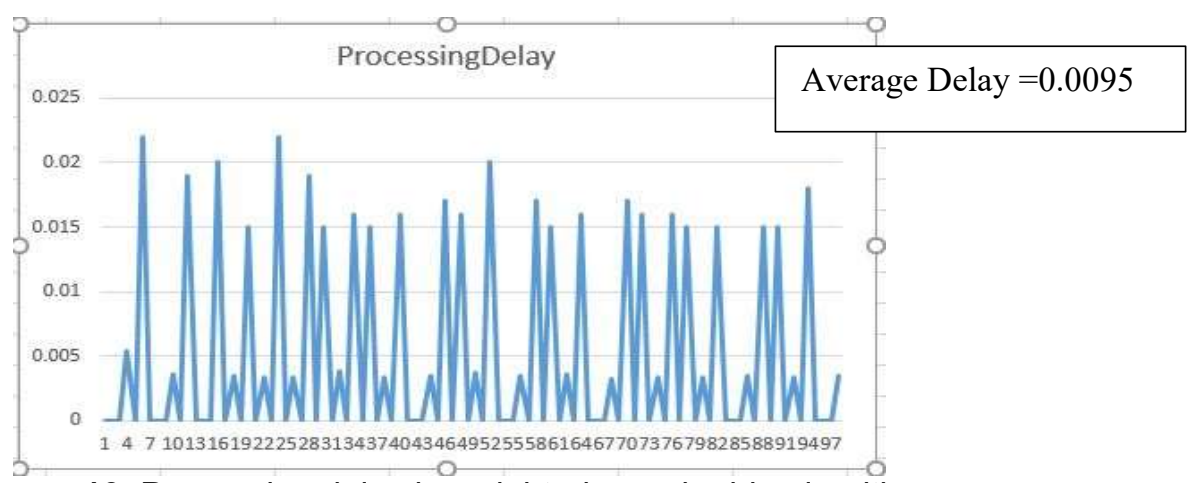

Figure 13. Processing delay in weighted round robin algorithm

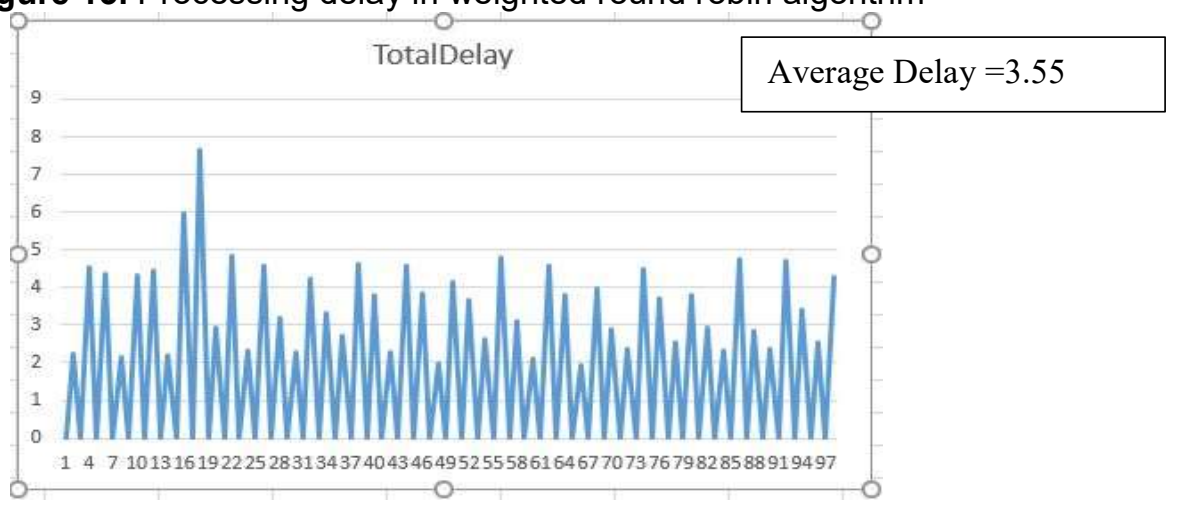

Figure 14. Total delay in weighted round robin algorithm

\section{Conclusion}

This paper shows the few conclusions based on the real time data. Here, processing time is taken during the some calculations on the fog as well as on the cloud. When data is collected on the fog node from the various sensors, some calculation takes the processing time. This shows the how many tickets have been scanned at what time and how many not. Presence of the ticket shows the presence of the passengers and vice versa.

The conclusion table (Table 1) is given in section 5 of this paper. This table is considering table only, not the real tested means not in railway coach. While, all the scenario is real implementation. Figure 1 shows the result with respect to Cloud environment, the time taken by the cloud averagely in a network are below 1.5 milliseconds while processing time in the cloud is approximately $0.02-0.03$ millisecond and the total time both of them is 1.4 milliseconds. Figure 6 shows that, the time taken in system is $15 \mathrm{~ms}$ to achieve cloud. While, the processing time in cloud is between $0.02-0.03 \mathrm{~ms}$, and the total time is averagely $14 \mathrm{~ms}$ Figure 7 shows that, network time is beneath $4 \mathrm{~ms}$ averagely. Processing time is about $1.4 \mathrm{~ms}$ averagely and total time is around $5 \mathrm{~ms}$. By analyzing these three figures we can say that in cloud total delay in Figure 1 is less than the total delay in local fog in Figure 5. So cloud may be the best solution for the requiring data, but when there is the decrement in the bandwidth up to $10 \mathrm{mbps}$ from $100 \mathrm{mbps}$, then fog is the solution for better response by comparing with Figure 3.Fog is not the total replacement of Cloud but it is the extended form of cloud computing. When we have implemented simple round robin algorithm and weighted round robin algorithm, we see that weighted round robin algorithm is better than all other algorithm implemented only in this paper. In the future, we plan to study an optimized (or near optimal) cooperation probability tuning algorithms. And want to merge least connection and least response time algorithm together.

Finally, we can conclude that if we implement the scenario like above, we can definitely increases the number of confirmed ticket. 
Table 1.Real Time Data on the fog and cloud

\begin{tabular}{|c|c|c|c|c|c|c|c|c|c|}
\hline $\begin{array}{l}\text { Train } \\
\text { Numb } \\
\text { er }\end{array}$ & $\begin{array}{l}\text { Tota } \\
\text { I } \\
\text { Seat } \\
\mathrm{s} \text { in } \\
\text { Trai } \\
\mathrm{n}\end{array}$ & $\begin{array}{l}\text { Total } \\
\text { Confirm } \\
\text { ed } \\
\text { Allotted } \\
\text { Seat }\end{array}$ & \multicolumn{3}{|c|}{$\begin{array}{l}\text { Total Seat Covered } \\
\text { By Passenger }\end{array}$} & \multicolumn{3}{|c|}{$\begin{array}{l}\text { Total uncovered } \\
\text { seat by passenger } \\
\text { due to any reason }\end{array}$} & $\begin{array}{l}\text { Seat Can } \\
\text { be given to } \\
\text { the } \\
\text { passenger } \\
\text { who are in } \\
\text { queue }\end{array}$ \\
\hline \multirow[t]{4}{*}{$\begin{array}{l}12345 \\
67\end{array}$} & \multirow{4}{*}{$\begin{array}{l}100 \\
\text { (say }\end{array}$} & \multirow[t]{4}{*}{$\begin{array}{l}100 \\
\text { (say) }\end{array}$} & $\begin{array}{l}\text { Cas } \\
\text { e } 1\end{array}$ & $\begin{array}{l}\text { Case } \\
2\end{array}$ & $\begin{array}{l}\text { Case } \\
3\end{array}$ & $\begin{array}{l}\text { Case } \\
1\end{array}$ & $\begin{array}{l}\text { Case } \\
2\end{array}$ & $\begin{array}{l}\text { case } \\
3\end{array}$ & \multirow[t]{4}{*}{2} \\
\hline & & & 100 & 0 & 0 & 0 & 0 & 0 & \\
\hline & & & 0 & 99 & 0 & 0 & 1 & 0 & \\
\hline & & & 0 & 0 & 99 & 0 & 0 & 1 & \\
\hline \multirow[t]{4}{*}{$\begin{array}{l}12345 \\
68\end{array}$} & \multirow{4}{*}{$\begin{array}{l}100 \\
\text { (say } \\
\text { ) }\end{array}$} & \multirow[t]{4}{*}{$\begin{array}{l}100 \\
\text { (say) }\end{array}$} & 100 & 0 & 0 & 0 & 0 & 0 & \multirow[t]{4}{*}{2} \\
\hline & & & 0 & 99 & 0 & 0 & 1 & 0 & \\
\hline & & & 0 & 0 & 99 & 0 & 0 & 1 & \\
\hline & & & 100 & 0 & 0 & 0 & 0 & 0 & \\
\hline \multirow[t]{4}{*}{$\begin{array}{l}12345 \\
69\end{array}$} & \multirow{4}{*}{$\begin{array}{l}100 \\
\text { (say }\end{array}$} & \multirow{4}{*}{$\begin{array}{l}100 \\
\text { (say) }\end{array}$} & 100 & 0 & 0 & 0 & 0 & 0 & \multirow[t]{2}{*}{2} \\
\hline & & & 0 & 99 & 0 & 0 & 1 & 0 & \\
\hline & & & 0 & 0 & 99 & 0 & 0 & 1 & \multirow{2}{*}{$\begin{array}{l}\text { Total } \\
\text { Seat }=2+2+ \\
2=6\end{array}$} \\
\hline & & & 100 & 0 & 0 & 0 & 0 & 0 & \\
\hline
\end{tabular}

The above table (Table 1) shows the three cases:

Case 1: it shows the $100 \%$ covered seat by passengers

Case2: it shows the seat covered by passenger at the source station due late of the passenger. Case3: it shows the seat covered by the passenger after leaving the source station and middle of the destination station.

So conclusion is that by applying the above approach we are getting the chances to get the blank seat that can be given to the others who are in waiting queues.

\section{References}

[1] Nur W. Rahayu; Dhery D. Andika (ICCOINS_2014. 6.3-5), "Assessing the Quality of Rail Ticket Reservation Systems: Cases from Indonesia" IEEE Xplore, pp 1-5, IEEE, June 2014. 
[2] Mohammad Aazam, Sherali Zeadally, Khaled A. Harras, "Offloading in Fog Computing for IoT: Review, Enabling Technologies, and Research Opportunities", Future Generation Computer Systems, volume-87, Pages 278-289, Elsevier, October 2018.

[3] Dastjerdi, A.V., Buyya, and R., "Fog computing: Helping the Internet of Things realizes its potential." IEEE Journals, Volume: 49, Issue: 8, Pages 112-116, August 2016.

[4] Inayat, Z.; Gani, A.; Anuar, N.B.; Anwar, S.; Khan, M.K.: "Cloud-based intrusion detection and response system: open research issues, and solutions", Arab. J. Sci. Eng., Volume: 42, Issue: 2, pp.399-423,Springer Berlin Heidelberg, 27 March 2017.

[5] Mohammad Aazam, Eui-Nam Huh, "Fog Computing: The Cloud-loT/loE Middleware Paradigm", IEEE Potentials, vol. 35, issue: 3, May 2016.

[6] Mohammad Aazam, Eui-Nam Huh, "Fog Computing and Smart Gateway Based Communication for Cloud of Things", in the proceedings of 3rd IEEE Future Internet and Cloud (FiCloud), Barcelona, Spain, pp: 464-470, 27-29 August, 2014.

[7] Gred Kortuem, Fahim kawsar, Daniel Fitton, and Vasughi Sundramoorthi, "Smart Objects and Building Blocks of Internet of Things", IEEE Internet Computing General, volume 14, issue 1, pp. 44-51, Jan.-Feb., 2010.

[8] Shuai Zhang et. Al., "Cloud Computing Research and Development Trend", in the proceedings of International Conference on Future Networks,IEEE Computer Society Washington, DC, USA, Pages 93-97, 22-24 Jan., 2010, Sanya China.

[9] Y. Jadeja, et. al., "Cloud Computing - Concepts - Concepts, Architecture and Challenges", in the proceedings of International Conference on Computing Electronics and Electrical Technologies, 21-22 March, 2012, Nagercoil, India.

[10] Jayavardhana Gubbi, Raj Kumar Buyya, Slaven Marusic, and Marimuthu Palaniswami, "Internet of Things (IoT): A Vision, Architectural Elements, and Future Directions", Technical Report CLOUDS-TR2012-2, July 2012.

[11]M. Jia, W. Liang, Z. Xu, and M. Huang, "Cloudlet load balancing inwireless metropolitan area networks," in INFOCOM 2016-The 35th Annual IEEE International Conference on Computer Communications, IEEE. IEEE, 2016, pp. 1-9.

[12]W. Yang, M. Hua, J. Zhang, T. Xia, J. Zou, C. Jiang, and M. Wang,“Enhanced system acquisition for nb-iot," IEEE Access, vol. 5, pp. 13 179-13 191, 2017.

[13]T. Takagi, "Reservation-Compulsory Commuting Railways: Innovation that will be Made Possible by UCRT/IPASS", Proceedings of COMPRAIL 2018 Conference, July 2018. 